\title{
The marching velocity of the capillary meniscus in a microchannel*
}

\author{
Lung-Jieh Yang ${ }^{1}$, Tze-Jung Yao ${ }^{2}$ and Yu-Chong Tai ${ }^{2}$ \\ ${ }^{1}$ Department of Mechanical and Electro-Mechanical Engineering, Tamkang University, 151, \\ Ying-Chuan Rd, Tamsui, 25137, Taiwan, Republic of China \\ ${ }^{2}$ Department of Electrical Engineering, California Institute of Technology, Pasadena, \\ CA 91125, USA
}

E-mail: ljyang@mail.tku.edu.tw

Received 19 March 2003, in final form 27 August 2003

Published 17 November 2003

Online at stacks.iop.org/JMM/14/220 (DOI: 10.1088/0960-1317/14/2/008)

\begin{abstract}
In this paper we describe an experimental method and an analytical model for characterizing the surface energy inside a microchannel of micrometer size by measuring the marching velocity or position of a capillary meniscus. This method is based on the fact that the force summation of the meniscus surface tension and the filling reservoir gravitation might produce a pressure to pull liquid into the channel, and the marching velocity or the instantaneous position of the meniscus is related to the surface energy. Both parylene and silicon-nitride microchannels with different surface conditions were fabricated to perform the fill-in experiments subject to different liquids. It is shown that our model agrees well with the experimental data and is a valid method.
\end{abstract}

\section{Introduction}

The capillary phenomenon was first studied in the early period of the 19th century. There has been almost no new progress since the 1920s due to the constraints of experimental facilities and techniques. Since microfluidics are involved, the topic of capillary meniscus is one of microscopic dimensions in classical mechanics which are still very important.

The capillary phenomenon can be defined quantitatively in terms of surface tension. Surface tension makes the surface of a liquid act as an elastic sheath, which minimizes the surface area of liquid so as to minimize the energy of the fluidic system. Although the liquid/solid contact angle method has been established for decades to measure the surface energy of a flat surface, to our knowledge there is no existing method to measure the surface properties inside a capillary tube or channel, especially in the micro domain. It is the goal of this work to develop an easy measurement method and we propose to use the marching velocity or position of the meniscus front to measure the surface energy inside a microchannel.

\footnotetext{
* This paper was presented at the 15th IEEE MEMS conference, held in Las Vegas, USA, 20-24 January 2002, and is an expansion of the abstract as printed in the Technical Digest of this meeting.
}

\section{Surface energy}

\subsection{Young's law}

Figure 1 shows a liquid droplet on a solid surface at steady state. There are three surface forces, including $\gamma_{\mathrm{la}}, \gamma_{\mathrm{sl}}$, and $\gamma_{\text {sa }}$, acting at the liquid/solid/air interface satisfying Young's law as [1-3]

$$
\gamma_{\mathrm{sa}}=\gamma_{\mathrm{sl}}+\gamma_{\mathrm{la}} \cos \theta_{\mathrm{C}}
$$

where $\theta_{\mathrm{C}}$ is the contact angle.

\subsection{Surface energy of a capillary microchannel}

From the energy perspective, the effective surface tension force applied on the fluid column can be deduced from the derivative of the surface energy of the whole fluidic system with respect to the spatial coordinate [4]. Figure 2 is the configuration of a capillary microchannel. The total surface energy of the capillary channel is composed of four parts. The first is the vacant area $\left(A_{T}-A_{X}\right)$ multiplied by $\gamma_{\mathrm{sa}}$. The second part is the wetting area $A_{X}$ multiplied by $\gamma_{\mathrm{sl}}$. The third part is the surface energy $E_{0}$ stored in the filling reservoir. $E_{0}$ hardly changes due to the infinitesimal amount of liquid filling into the capillary. The fourth part is the complex surface of capillary 


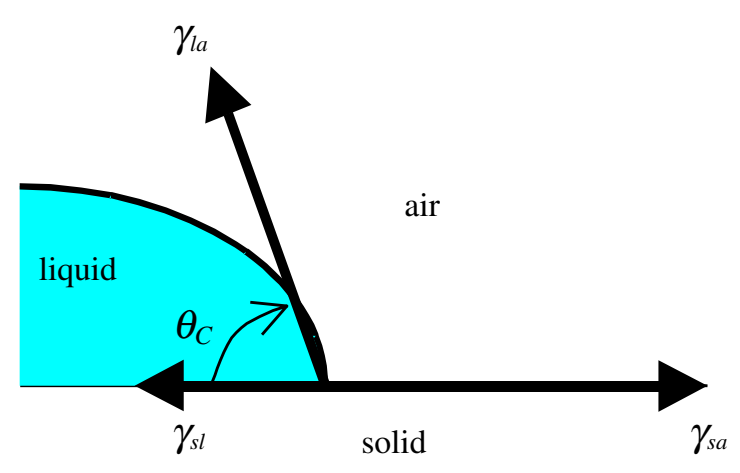

Figure 1. The liquid/solid/air interface of a droplet. $\theta \mathrm{c}$ is the contact angle and $\gamma_{\mathrm{ij}}$ denotes surface tension between phase $i$ and $j$.

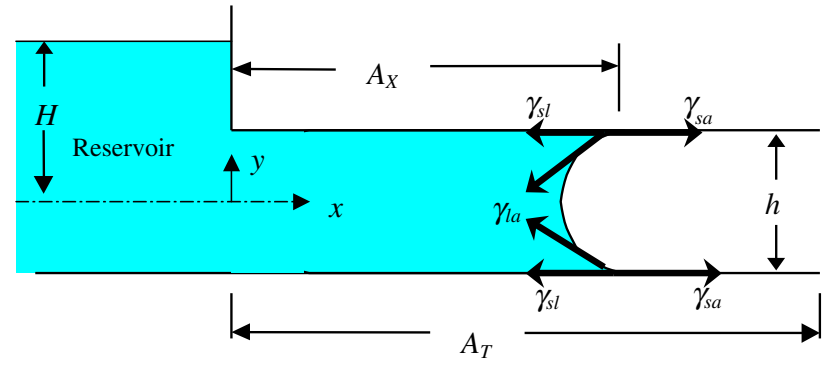

Figure 2. The configuration of a microchannel. $h$ is the channel height. $A_{x}$ denotes wetting area with the length of $x$, and $A_{T}$ denotes the total area with the length of $l$. The liquid reservoir is assigned at the very left of this figure. $H$ denotes the height of the liquid reservoir above the centerline of the capillary channel.

meniscus front multiplied by $\gamma_{\text {la }}$. We neglect the fourth term because of the very small area of meniscus front compared to other surfaces.

Then the total energy of the capillary channel in figure 2 is expressed as

$$
E_{S}=E_{0}+\left[A_{T} \gamma_{\mathrm{sa}}+A_{X}\left(\gamma_{\mathrm{sl}}-\gamma_{\mathrm{sa}}\right)\right]
$$

Assuming that the cross-section of the capillary channel in figure 2 is rectangular with a width of $w$ and height of $h$, the total energy can be expressed as

$$
E_{S}=E_{0}+2(h+w)\left[l \cdot \gamma_{\mathrm{sa}}-x\left(\gamma_{\mathrm{sa}}-\gamma_{\mathrm{sl}}\right)\right]
$$

\subsection{Capillary force}

Taking the derivative of equation (3) with respect to $x$, we obtain the equivalent capillary force $F_{S}$ applied on the fluid column along the $x$-direction:

$$
F_{S}=-\frac{\mathrm{d} E_{S}}{\mathrm{~d} x}=2(h+w) \cdot\left(\gamma_{\mathrm{sa}}-\gamma_{\mathrm{sl}}\right)=\Delta p_{\mathrm{la}} \cdot w \cdot h
$$

The pressure drop $\Delta p_{\text {la }}$ across the liquid-air interface is therefore deduced under the assumption that channel height $h$ is much smaller than channel width $w$ [5]:

$$
\Delta p_{\mathrm{la}}=\frac{2(h+w)\left(\gamma_{\mathrm{sa}}-\gamma_{\mathrm{sl}}\right)}{w h} \approx \frac{2\left(\gamma_{\mathrm{sa}}-\gamma_{\mathrm{sl}}\right)}{h}
$$

Equation (5) can be rewritten as the so-called 'Laplace pressure drop' for the capillary tube by replacing the hydraulic radius $r_{h}\left(=D_{h} / 2=w h /(w+h)\right)$ of the rectangular microchannel with the inner radius symbol $r$ of capillary tube:

$$
\Delta p_{\mathrm{la}}=\frac{2\left(\gamma_{\mathrm{sa}}-\gamma_{\mathrm{sl}}\right)}{r}=\frac{2 \cdot \gamma_{\mathrm{la}} \cdot \cos \theta_{\mathrm{C}}}{r}
$$

Equation (5) or Laplace pressure (6) demonstrate that the smaller the channel/capillary tube dimension, the larger the pressure drop across the liquid-air capillary interface.

\subsection{The measurement of contact angles}

Contact angle measurement is a powerful way to study the properties of open surfaces. However, measuring the inner surface properties is much more difficult. Sobolev et al [6] tried to correlate the pumping pressure, the position or the velocity and the contact angle of a meniscus in a quartz capillary tube using a delicate, image-capturing apparatus. Such a measurement of the inner surface properties of a microchannel is very important for microfluidics and bioMEMS.

However, the relation between the meniscus position and the filling time was originally found in the experiments with horizontal capillary tubes wetted by liquids in 1902 and 1921 $[7,8]$. Therefore, in this paper we aim to measure the marching position and velocity of a capillary meniscus in micromachined channels with the dimension of less than $100 \mu \mathrm{m}$ to interpret microfluidic properties. Experimentally, filling water into a microchannel automatically with different surface conditions can generate a variety of marching velocities for the same liquid. In section 3 we describe the theoretical construction of a one-dimensional mathematic model, which is based on the force balance between the momentum of the channel flow, filling-reservoir gravitation and the meniscus (Laplace) pressure of figure 2 .

\section{The motion model of a marching meniscus}

Once the pressure drop of the meniscus front is known, we can use the incompressible Navier-Stokes equation to derive the motion solution of the capillary meniscus front in figure 2 . Treated as a one-dimensional time-variant fluid field with only the horizontal velocity variable $u$ generally varying with the spatial coordinates and time, the instantaneous position of capillary meniscus $L(t)$ is described as the following differential system:

continuity equation (conservation of mass)

$$
\frac{\partial u}{\partial x}=0 ; \quad(\Rightarrow u=u(y, t))
$$

momentum equation (conservation of momentum)

$$
\begin{gathered}
\frac{\partial u}{\partial t}=-\frac{1}{\rho} \frac{\mathrm{d} p}{\mathrm{~d} x}+\frac{\mu}{\rho} \frac{\partial^{2} u}{\partial y^{2}} \\
-\frac{\mathrm{d} p}{\mathrm{~d} x}=\frac{1}{L(t)}\left[\rho \cdot G \cdot H+\frac{2\left(\gamma_{\mathrm{sa}}-\gamma_{\mathrm{sl}}\right)}{h}\right]
\end{gathered}
$$

position of meniscus

$$
u(0, t)=\frac{\mathrm{d} L(t)}{\mathrm{d} t}
$$

boundary condition

$$
\begin{gathered}
u\left(\frac{h}{2}, t\right)=0 \quad \text { (non-slip boundary condition) } \\
\frac{\partial u}{\partial y}(0, t)=0 \quad \text { (symmetric along the centerline) }
\end{gathered}
$$


initial condition

$$
u(y, 0)=0 ; \quad L(0)=L_{0}
$$

Here, $G$ denotes the gravitation acceleration and $H$ denotes the height of liquid reservoir above the center of the capillary channel in equation $(8 a)$. Equation $(8 a)$ also reveals that the external pressure or force ready to accelerate or motivate the liquid column into the channel is not only the capillary force but also the gravitational effect from the filling liquid reservoir [8]. On the other hand, the exact velocity variation along the $y$-direction is of minor concern in this model. We substitute a parabolic distribution equation (13) of $u(y, t)$, which satisfies the non-slip boundary condition (10) and the symmetric condition (11) automatically, into equation (8):

$$
u(y, t)=L^{\prime}(t) \cdot\left[1-\left(\frac{2 y}{h}\right)^{2}\right]
$$

Then we obtain equation (14):

$$
\begin{aligned}
& L^{\prime \prime}(t) \cdot\left[1-\left(\frac{2 y}{h}\right)^{2}\right]+\frac{\mu}{\rho} \frac{8}{h^{2}} \cdot L^{\prime}(t) \\
& =\frac{\rho \cdot G \cdot H+2\left(\gamma_{\mathrm{sa}}-\gamma_{\mathrm{sl}}\right) / h}{\rho \cdot L(t)}
\end{aligned}
$$

Equation (14) basically demonstrates the force balance between the momentum of channel flow and the pressure drop from the reservoir gravitation as well as the meniscus capillary effects. It will be inherently reasonable if we additionally take the average of equation (14) along the $y$-direction. Then the initial value problem (IVP) of the marching position $L(t)$ of the capillary meniscus with respect to time is

$\left(L^{\prime \prime}+\frac{12 \mu}{\rho h^{2}} L^{\prime}\right) \cdot L=\frac{3}{2 \rho}\left[\rho \cdot G \cdot H+\frac{2\left(\gamma_{\mathrm{sa}}-\gamma_{\mathrm{sl}}\right)}{h}\right]$

Initial Condition : $L^{\prime}(0)=0 ; \quad L(0)=L_{0}$

Since there is no exact solution of the nonlinear IVP, equations (15) and (16), we have to solve it numerically [9]. However, if we neglect the acceleration (second derivative) term of equation (15) that is the case in our time domain $(t \gg \mu \mathrm{s})$, then the simplified asymptotic solution of $L(t)$ can be regarded as the marching position following a square-root relation with time.

$$
L(t)=\sqrt{L_{0}^{2}+D t} ; \quad D=\frac{h^{2}}{4 \mu}\left[\rho \cdot G \cdot H+\frac{2\left(\gamma_{\mathrm{sa}}-\gamma_{\mathrm{sl}}\right)}{h}\right]
$$

where $D$ is characterized as a diffusing coefficient in this problem.

\section{Results and discussion}

\subsection{The parylene microchannels}

Experimentally, we fabricated a silicon chip with parylene microchannels as shown in figures $3(a)$ and $(b)$ to perform the water-filling experiment [10]. The parylene channels were designed with the widths of $10,20,40$ and $80 \mu \mathrm{m}$. The height of the parylene microchannel is determined by the thickness of the sacrificial photoresist. In this work, the AZ4620 positive resist is assigned as the sacrificial layer to construct the $3 \mu \mathrm{m}$ high channel. For the thickness variation of
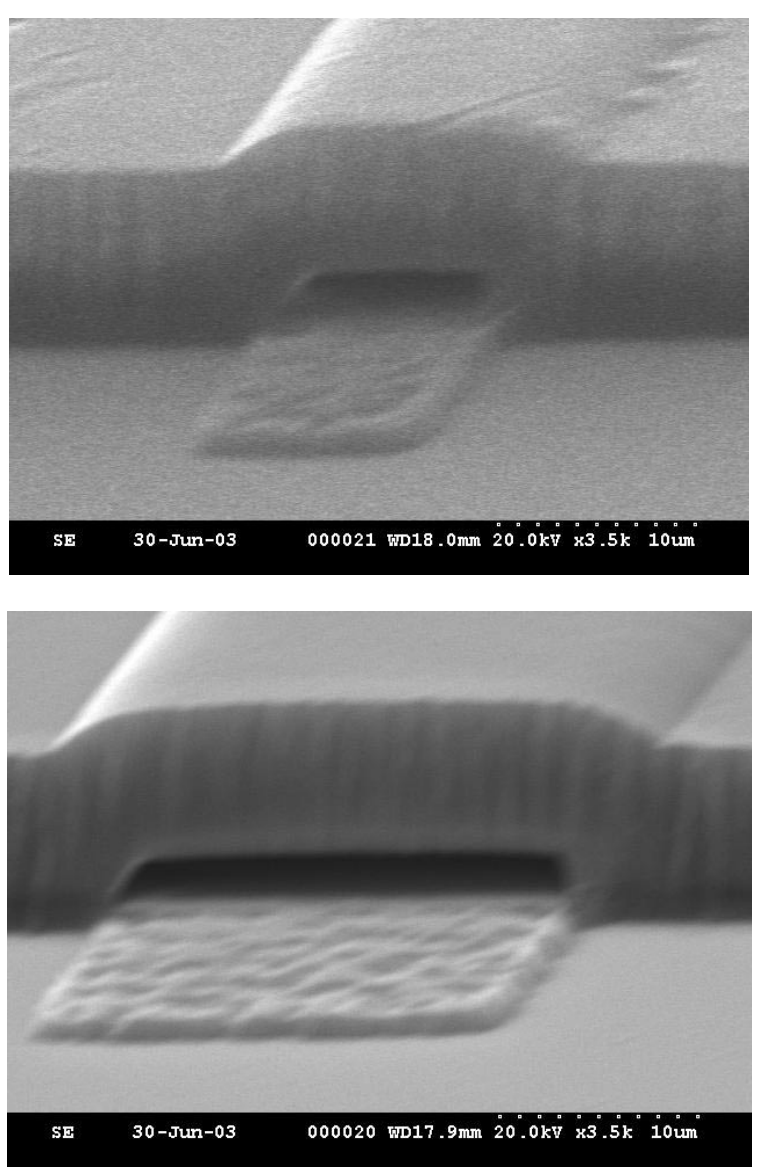

Figure 3. SEM photographs of the parylene channels, viewing the entrance of the microchannels with the height of $2.6 \mu \mathrm{m}$. (a) $10 \mu \mathrm{m}$ wide channel; (b) $20 \mu \mathrm{m}$ wide channel.

the wafer-level process, we in fact confirm the channel height as $2.6 \mu \mathrm{m}$ in figure 3(a) using scanning electron microscopy (SEM). The non-uniformity of the sacrificial photoresist along a certain parylene microchannel is locally below $5 \%$ measured by an Alpha-Step-50 surface profiler. Additionally, the local tapering of the rectangular cross-section of the parylene mirochannel shown in figure 3 did not apparently influence the global capillary force. The reason is that the width-to-height ratios of the parylene channels here were designed from 3.3 to 26.7 on purpose to minimize the sidewall effect. Such a channel design shows itself to be a good experiment platform to prove the one-dimensional mathematical model useful in the following sections.

\subsection{General observation}

The moving images of water meniscus in the parylene channel, as shown in figure 4, subjected to dry, pre-wetted and pretreated (with agents other than water) inner walls, were recorded through an optical microscope and a CCD onto a VCR tape. We transformed the VCR images into digital movie files, which have a time resolution of $1 / 30 \mathrm{~s}$. Finally the information regarding the position of the moving meniscus was collected and this is plotted in figure 5. Fitting the data according to the square-root relation in equation (17) by the least-squares error method, we obtained the diffusing coefficients $D$ as well as the surface energies $\left(\gamma_{\mathrm{sa}}-\gamma_{\mathrm{sl}}\right)$ in table 1. 


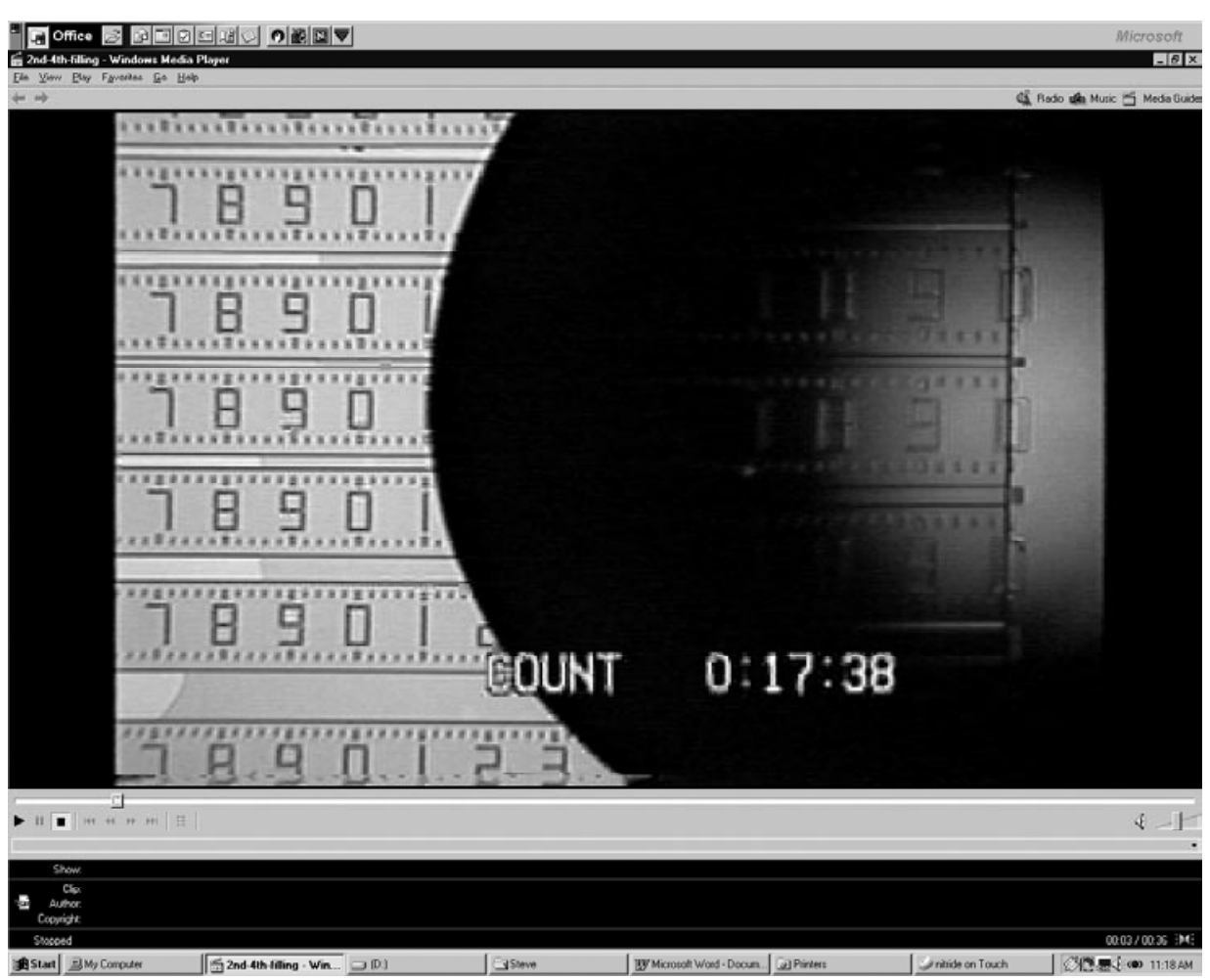

Figure 4. The water-filling view of parylene channels. $2 \mathrm{~mm}$ long, $2.6 \mu \mathrm{m}$ high, and 80, 40, 20 and $10 \mu \mathrm{m}$ wide (from bottom to top). Each small step of the ruler is $20 \mu \mathrm{m}$. The water droplet (dark area) serves as a reservoir at the right inlet.

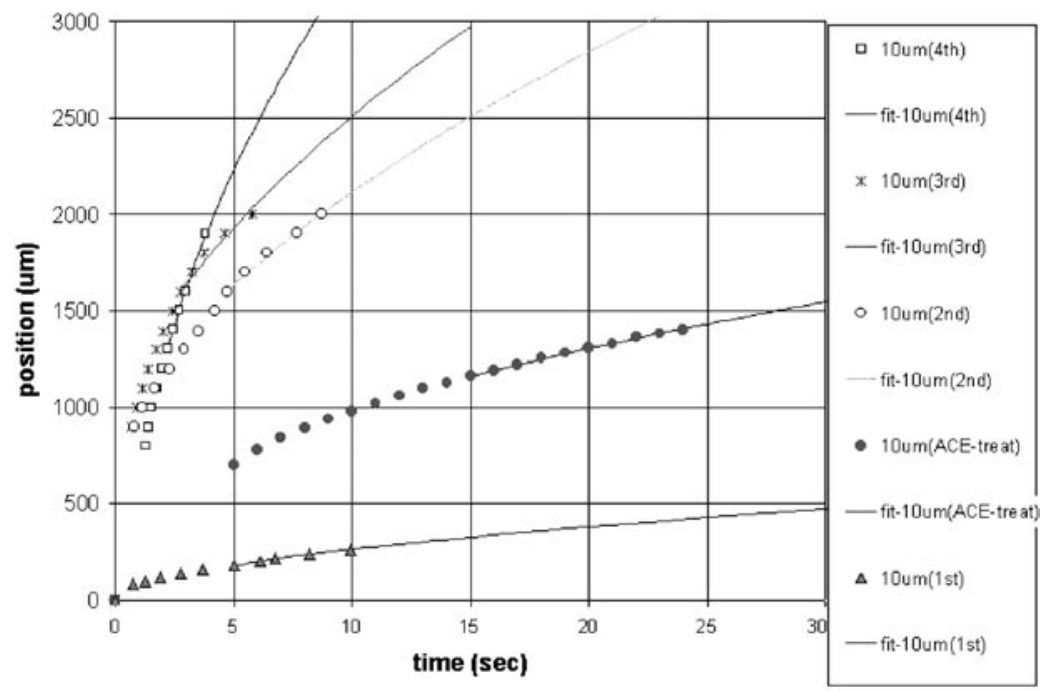

Figure 5. The position of a capillary meniscus versus time of deionized water filling into a parylene channel. The fitting curves denote the theoretical modeling of equation (17).

Clearly, the square-root dependence is valid and this model fits with the experimental data really well in figure 5 . The results shown in table 1 reveal very small values of the surface energy difference $\left(\gamma_{\mathrm{sa}}-\gamma_{\mathrm{sl}}\right)$ compared to the surface energy of water $\left(\gamma_{1 \mathrm{a}}=0.073 \mathrm{Nt} \mathrm{m}^{-1}\right)$. In other words, this work obtained the dynamic contact angles indirectly using Young's law or equation (1) by knowing the surface energy $\left(\gamma_{\mathrm{sa}}-\gamma_{\mathrm{sl}}\right)$ and $\gamma_{\mathrm{la}}$ in advance. For example, the deduced dynamic contact angles $\theta_{\mathrm{C}}$ for different cases of water meniscus of a parylene microchannel in table 1 are slightly smaller or greater than $90^{\circ}$, which is close to the static contact angle measurement of $86 \pm 3^{\circ}$ done on the clean, planar parylene surface.

\subsection{The gravitational pumping effect from the liquid reservoir}

Why does the water-filling phenomenon still occur in a capillary channel with (slightly) hydrophobic inner surface (case B of table 1)? It may be clarified by the diffusing coefficients $D$ in equation (17). Two factors including surface tension and gravitational forces contribute to the water-filling 


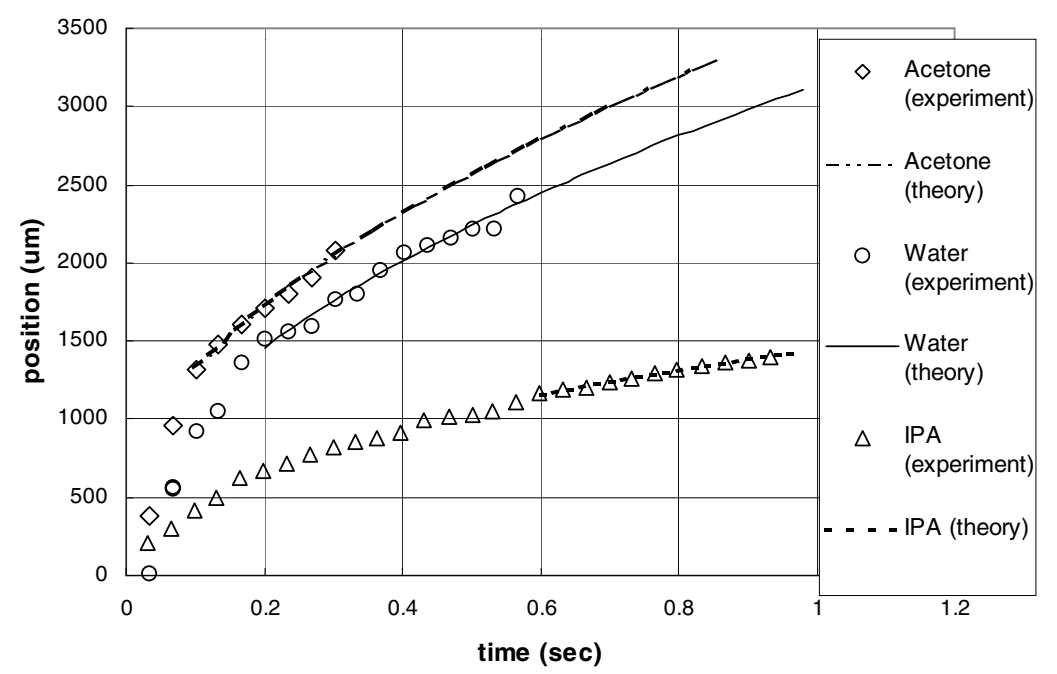

Figure 6. The position of a capillary meniscus versus time of different liquids filling into a silicon-nitride microchannel.

Table 1. The flow parameters extracted from the data of the water-filling experiment in figure 5. (The channel height $h$ is $2.6 \mu \mathrm{m} \pm 5 \%$, the viscosity for water is $0.00108 \mathrm{Nt} \mathrm{s} \mathrm{m}^{-2}$, and $\rho \cdot G \cdot H$ is assumed as $10 \mathrm{Nt} \mathrm{m}^{-2} \pm 10 \%$.)

\begin{tabular}{lccrl}
\hline & $\begin{array}{l}\text { Diffusing coefficient } D \\
\left(\mu \mathrm{m}^{2} \mathrm{~s}^{-1}\right)\end{array}$ & $\begin{array}{l}{\left[\rho \cdot G \cdot H+\frac{2\left(\gamma_{\mathrm{sa}}-\gamma_{\mathrm{sl}}\right)}{h}\right]} \\
\left(\mathrm{Nt} / \mathrm{m}^{2}\right)\end{array}$ & $\begin{array}{l}\gamma_{\mathrm{sa}}-\gamma_{\mathrm{sl}}=\gamma_{\mathrm{aa}} \cos \theta_{\mathrm{C}} \\
(\mathrm{Nt} / \mathrm{m})\end{array}$ & $\theta_{\mathrm{C}}($ degree $)$ \\
\hline $\mathrm{A}$ & 68450 & $44(40 \sim 54)$ & $0.00004 \pm 20 \%$ & $90.0 \pm 0.01 \%$ \\
$\mathrm{~B}$ & 7584 & $5(4 \sim 6)$ & $-0.00001 \pm 50 \%$ & $90.0 \pm 0.01 \%$ \\
$\mathrm{C}$ & 361500 & $231(210 \sim 285)$ & $0.00029 \pm 12 \%$ & $89.9 \pm 0.02 \%$ \\
$\mathrm{D}$ & 514300 & $329(298 \sim 406)$ & $0.00041 \pm 12 \%$ & $89.7 \pm 0.03 \%$ \\
$\mathrm{E}$ & 1177000 & $752(682 \sim 929)$ & $0.00096 \pm 12 \%$ & $89.2 \pm 0.09 \%$ \\
\hline
\end{tabular}

(A) Pre-treated with acetone for three times before filling water.

(B) 1 st time of filling water after stripping photoresist (dry capillary).

(C) 2nd time of filling water (pre-wetted capillary).

(D) 3rd time of filling water.

(E) 4th time of filling water.

motion simultaneously. For the general case of microchannels with high surface energies, the gravitation term $\rho \cdot G \cdot H$ is usually much smaller than the surface tension term $\frac{2\left(\gamma_{\mathrm{sa}}-\gamma_{\mathrm{sl}}\right)}{h}$. Then surface tension dominates the water-filling behavior in hydrophilic microchannels, e.g., quartz tubes or silicon-nitride channels. However, for the microchannels made by parylene or other polymer materials, the gravitational force $\rho \cdot G \cdot H$ may intrinsically turn out to have the same order of magnitude with low surface energy $\frac{2\left(\gamma_{\mathrm{sa}}-\gamma_{\mathrm{sl}}\right)}{h}$. The gravitational pumping from the liquid reservoir may still push the capillary meniscus forward even encountering a hydrophobic dragging from the surface tension effect $\left(\frac{2\left(\gamma_{\mathrm{sa}}-\gamma_{\mathrm{sl}}\right)}{h}=\frac{2 \gamma_{\mathrm{a}} \cos \theta_{\mathrm{C}}}{h}<0\right.$ !).

A liquid droplet was dispensed at the entrance of the microchannels by a pipette as the liquid reservoir in this work. The radius of the liquid droplet is measured as $1 \mathrm{~mm} \pm 10 \%$ directly by the optical microscope.

\subsection{The local collapse of upper walls of parylene channels}

Reconsidering the almost identical values of the dynamic contact angles in table 1, the physical possibility of this result may be questioned. We therefore prepared some samples of parylene surfaces with the same pretreatment as the parylenechannel chip, and measured the contact angles once again. (The pretreatment is shown as follows: coating photoresist $\rightarrow$ soft-baking at $90{ }^{\circ} \mathrm{C} \rightarrow$ stripping photoresist in acetone for
$24 \mathrm{~h}$.) The contact angles of the pretreated, planar parylene surfaces were found to be $75 \pm 2^{\circ}$, which are much smaller than the predicted values in table 1 .

Based on the claim of hydrophilic capillary microchannels with high surface energies herein, one interesting explanation for the very low speed of the filling flow is that a local collapse of the upper walls of the parylene channels occurred. The channel collapse is due to the strong meniscus pulling-down force, which overcomes the weak mechanical stiffness of the parylene channel walls. This tremendous shrinkage of the cross section area indeed increases the viscous drag and decreases the marching velocity.

If we assumed that the collapsed channel height $h$ is only $0.1 \mu \mathrm{m}$, the surface energies in table 1 are not small at all and the deduced dynamic contact angles range from $70^{\circ}$ to $89^{\circ}$ at the same time. On the contrary, if we assumed the dynamic contact angle is around $75^{\circ}$, the deduced channel height $h$ is from $0.001 \mu \mathrm{m}$ to $0.135 \mu \mathrm{m}$, which is matched with the postulated situation of channel-collapse!

\subsection{The huge variation of the meniscus velocities due to different surface states}

This water-filling observation also shows the importance of sample preparation when conducting experiments on polymer surfaces. The diffusing coefficient $D$ in fact changes by over 
Table 2. The flow parameters extracted from the data of the liquid-filling experiment in figure 6. (The silicon-nitride channels are subjected to piranha cleaning prior to the testing. The channel height $h$ is $0.5 \mu \mathrm{m} \pm 3 \%$. The viscosity data of acetone and IPA are both assumed to be $0.001 \mathrm{Nt} \mathrm{s} \mathrm{m}^{-2} \cdot \rho \cdot G \cdot H$ is assumed to be $10 \mathrm{Nt} \mathrm{m}^{-2} \pm 10 \%$.)

\begin{tabular}{|c|c|c|c|c|}
\hline $\begin{array}{l}\text { Filling } \\
\text { Liquid }\end{array}$ & $\begin{array}{l}\text { Diffusing coefficient } \\
D\left(\mu \mathrm{m}^{2} \mathrm{~s}^{-1}\right)\end{array}$ & $\begin{array}{l}{\left[\rho \cdot G \cdot H+\frac{2\left(\gamma_{\mathrm{sa}}-\gamma_{\mathrm{ss}}\right)}{h}\right]} \\
\left(\mathrm{Nt} \mathrm{m}^{-2}\right)\end{array}$ & $\begin{array}{l}\gamma_{\mathrm{sa}}-\gamma_{\mathrm{sl}}=\gamma_{\mathrm{la}} \cos \theta_{\mathrm{C}} \\
(\mathrm{Nt} / \mathrm{m})\end{array}$ & $\theta_{\mathrm{C}}($ degree $)$ \\
\hline Acetone & 12061200 & $192979 \pm 6 \%$ & $0.0482 \pm 3 \%$ & Not available \\
\hline DI water & 9656500 & $166864 \pm 6 \%$ & $\pm 3 \%$ & $55.2 \pm 2 \%$ \\
\hline IPA & 1936595 & $30986 \pm 6 \%$ & $0.0078 \pm 3 \%$ & Not available \\
\hline
\end{tabular}

three orders of magnitude in table 1 . The change in parylene surface energy with different processes is a well-known and intricate phenomenon. Processes such as photoresist spinning and stripping, plasma roughening and solvent immersion can change the parylene surface to a great extent.

The large variation of diffusing coefficients $D$ and the surface energies $\left(\gamma_{\mathrm{sa}}-\gamma_{\mathrm{sl}}\right)$ in table 1 can be explained by surface adsorption of water molecules inside the parylene microchannel. Right after the first filling and drying, the inner parylene surface adsorbs water molecules, which allow the following water filling easier with a much higher (two order of magnitude) speed. This hypothesis is supported by the fact that the marching velocity will reverse back to the initial water filling in table 1 if we treat the inner channel surface with acetone again (same as the channels were prepared after stripping away the sacrificial photoresist).

\subsection{The filling experiment on the silicon-nitride microchannel}

To further test the theory developed for the liquid-filling experiment, $\mathrm{Si}_{x} \mathrm{~N}_{y}$ microchannels are also fabricated using $0.5 \mu \mathrm{m}$ polysilicon as a sacrificial layer. The thickness non-uniformity of polysilicon is well below 3\%. The sacrificial polysilicon is finally etched away using tetra methyl ammonium hydroxide (TMAH) and the channels are also subjected to piranha cleaning prior to the testing. The result of the liquid-filling experiment is shown in figure 6. One example of the diffusing coefficients extracted from figure 6 shows a high surface energy (difference) of $0.0417 \mathrm{Nt} \mathrm{m}^{-1}$. This value corresponds to the dynamic contact angle of $55.2^{\circ}$ $\pm 2 \%$, which is close to the static contact angle measurement of $45 \pm 5^{\circ}$ done on the clean, planar silicon-nitride surface. The extracted diffusing coefficients and the surface energies of a nitride channel using agents of acetone and IPA are also shown in table 2 .

Thus, the fill-in experiment herein not only describes the surface tension driven mechanism but also the surface state of a microchannel. It may inspire another possibility for identifying the microchannel with different generic parameters other than the geometric specification (size, roughness, etc.) on a micrometer scale. In other words, by performing the fill-in experiment of the working liquid and recording the instantaneous position of capillary meniscus, more precise information can be gathered to identify microfluidic parameters for applications such as microcooling and microinjection.

\section{Conclusion}

In this paper we introduce a method to measure the surface energy difference by monitoring the capillary meniscus in a microchannel. A one-dimensional mathematical model was concurrently proposed tracing the liquid-filling behavior and extracting the information of surface energies. Parylene and silicon-nitride microchannels were fabricated by surface micromachining technology to perform the liquid-filling experiment and prove the model valid. The measured data subjected to different surface states vary across three orders of magnitude in the case of the parylene microchannel. This also reveals that the gravitational force as well as the surface tension-induced collapse of the channel walls cannot be neglected in analyzing the filling flow of polymer microchannels. With the experimental methodology developed in this work, the mechanism of the surface tension driven flow becomes more apparent and can be applied to fluid delivery of microfluidic systems. Further development of this technique will facilitate collection of essential surface property data for further understanding of the microchannel flow.

\section{Acknowledgments}

The authors want to thank Mr Ken Walsh, Dr Xing Yang and $\mathrm{Dr}$ Yong $\mathrm{Xu}$ of Caltech Micromachining Laboratory for technical support. Help from Mr Yu-Lin Huang, Mr Jiun-Min Wang, Miss Hung-Yin Chen, Mr Shin-Shong Wang and Mr Po-Chiang Yang of Tamkang University is also highly appreciated. This project is financially supported by the NSF Engineering Research Center for Neuromorphic Systems Engineering (CNSE) at Caltech and the National Science Council of Taiwan ROC with the project number of NSC89-2217-E-032-001.

\section{References}

[1] Madou M 1997 Fundamentals of Microfabrication (New York: CRC Press) p 433

[2] Israelachvili J N 1985 Intermolecular and Surface Forces (London: Academic) p 120

[3] de Gennes P G 1985 Wetting: statics and dynamics Rev. Mod. Phys. 57 827-90

[4] Tas N et al 1996 Stiction in surface micromachining J. Micromech. Microeng. 6 385-97

[5] Tas N et al 2002 Nanofluidic bubble pump using surface tension directed gas injection Anal. Chem. 74 2224-7

[6] Sobolev V D 2000 J. Colloid Interface Sci. 222 51-4

[7] Bell J M and Cameron F K 1906 The flow of liquids through capillary spaces J. Phys. Chem. 10 658-74

[8] Washburn E W 1921 The dynamics of capillary flow Phys. Rev. 17 273-83

[9] O’Neil P V 1995 Advanced Engineering Mathematics (Pacific Grove: Brooks/Cole Publishing Company) p 773

[10] Walsh K et al 2001 Photoresist as a sacrificial layer by dissolution in acetone IEEE Proc. MEMS'O1 pp 114-7 